\title{
Orthogeriatric Nursing
}

\author{
Julie Santy-Tomlinson, Karen Hertz, \\ and Magdalena Kaminska
}

Nursing is central to good care for the patient with a fragility fracture and makes a major contribution to positive outcomes. Nurses are the largest group of health professionals in the orthogeriatric team and they are the one group who are present for the full 24-hour span during hospitalisation. They are also most likely to work across organisational boundaries, acting as links between the patient's home and local community, the hospital, the outpatient/ambulatory setting and other organisations.

Nurses who work in the orthogeriatric setting must be able to clearly articulate their role and value so that they can inform patients, their families and other members of the MDT what to expect from them. No single healthcare profession can provide care to fragility fracture patients in isolation, but it is known that patients' outcomes are improved if there is full collaboration across all disciplines making up the "orthogeriatric" team [1] and patients with a fragility fracture have numerous complex care needs that need a team approach that includes skilled, compassionate nursing. Although this chapter is concerned with orthogeriatric nursing generally, it is impossible to ignore the fact that of all fragility fractures, hip fracture is the most significant injury: it is the most common reason for admission to an orthopaedic ward, accounts for much orthopaedic bed occupancy and is a large portion of the

\footnotetext{
J. Santy-Tomlinson $(\square)$

Faculty of Biology, Medicine and Health, Division of Nursing, Midwifery and Social Work, School of Health Sciences, The University of Manchester, Manchester, UK

e-mail: Julie.santy-tomlinson@manchester.ac.uk

K. Hertz

Specialised Division, University Hospital of North Midlands,

Stoke-on Trent, Staffordshire, UK

e-mail: Karen.hertz@uhnm.nhs.uk

M. Kaminska

Faculty of Health Sciences, Department of Primary Health Care,

Pomeranian Medical University, Szczecin, Poland
} 
total cost of all fragility fractures. It is also the most expensive fracture in terms of volume and unit costs. Complexity of patient needs, prevalence, number of bed days and cost means that the focus of inpatient care tends to relate predominantly to this category of injury. However, the principal skills and knowledge needed to look after hip fracture patients well must be applied across the management of all older patients with fractures and include all fundamental aspects of nursing care for the adult as well as highly specialised interventions for older people.

The aim of this chapter is to consider the nature of orthogeriatric nursing and to explore its theoretical, political and professional aspects.

\subsection{Learning Outcomes}

At the end of the chapter, and following further study, the nurse will be able to:

- Explain the nature of orthogeriatric nursing, using adult and geriatric nursing theory and philosophy

- Explore the professional, ethical, legal and political aspects of orthogeriatric nursing

- Articulate the value of orthogeriatric nursing in achieving good outcomes for patients

- Discuss the importance of skill, knowledge and education in providing effective care to patients with fragility fractures.

\subsection{Nursing}

Nursing is broad and complex, so defining nursing enables nurses to explain to patients, families and others what they can expect from them. The ICN [2] defines both "nursing" and "a nurse" (see Box 12.1) to highlight the expanse of the nursing role across the entire lifespan, in all communities and with people with all healthcare needs. These definitions help to illuminate some of the central aspects of nursing care

Box 12.1: International Council of Nurses (ICN) Definitions of Nursing and a Nurse [1]

Definition of Nursing (Short Version)

"Nursing encompasses autonomous and collaborative care of individuals of all ages, families, groups and communities, sick or well and in all settings. Nursing includes the promotion of health, prevention of illness, and the care of ill, disabled and dying people. Advocacy, promotion of a safe environment, research, participation in shaping health policy and in patient and health systems management, and education are also key nursing roles". 


\section{Definition of a Nurse}

"The nurse is a person who has completed a program of basic, generalized nursing education and is authorized by the appropriate regulatory authority to practice nursing in his/her country. Basic nursing education is a formally recognized program of study providing a broad and sound foundation in the behavioral, life, and nursing sciences for the general practice of nursing, for a leadership role, and for post-basic education for specialty or advanced nursing practice. The nurse is prepared and authorized (1) to engage in the general scope of nursing practice, including the promotion of health, prevention of illness, and care of physically ill, mentally ill, and disabled people of all ages and in all health care and other community settings; (2) to carry out health care teaching; (3) to participate fully as a member of the health care team; (4) to supervise and train nursing and health care auxiliaries; and (5) to be involved in research".

that include activities related to the scope of nursing; how care is given; what knowledge, skills and education are needed and how nursing constitutes a profession.

Nursing is both a caring art and a science and it encompasses a distinct body of knowledge, separate from that of medical or allied health professional colleagues. Knowledge is specific information about something and caring is behaviour that demonstrates compassion and respect for another, but these simplified concepts do not truly reflect the synthesis of both knowledge and the art of caring that makes orthogeriatric nursing unique [3].

\subsection{The Orthogeriatric Patient}

Patients with fragility fractures present across the spectrum of health service providers including general practice, community services, acute care services including emergency portals, operating departments, inpatient and outpatient services and hospital- or community-based rehabilitation services. Care takes place in an environment that is largely not conducive to the care of frail older people. The provision of safe, effective care for hospitalised patients following hip fracture is particularly complex and demands a focus on achieving best outcomes for frail elderly hospitalised patients. This complexity is generated by multiple interlocking problems related to both breadth (range) and depth (severity) of healthcare need [4] related to three main characteristics; the person, the fracture and the care environment-all of which have a significant impact on patient care outcomes [5]. Orthogeriatric patients have usually fallen, often have multiple co-morbidities and are frequently frail. These problems interact in the aftermath of fragility fracture to increase care needs because of the increased physiological demands of the pre-, peri- and post-operative recovery and rehabilitation phases of care. 


\subsection{Care Quality}

Nursing is underpinned by a set of core personal and professional values, and the meaning of "quality care" varies depending on whether it is viewed from the perspective of the care giver or the care receiver but tends to be based on six core elements: a holistic approach, patient empowerment, professional accountability, patient safety, integrated teamwork and efficiency and effectiveness [6].

Compassion is a quality that enables nurses to be motivated to provide effective, person-centred care and is essential to quality nursing care; it includes empathy, respect and dignity, qualities that require understanding and recognition of another's suffering and a desire to do something about it. It is these qualities that enable nurses to see every patient as an individual and to humanise their care [7]. This is always important, but particularly when those receiving care are vulnerable as in the case of an elderly frail person who has suffered a hip fracture.

Quality care is significantly affected by communication, but even basic communication with vulnerable older adults accessing healthcare services is complex. Problems between individuals, families, caregivers and health professionals usually occur simply because communication is not effective. Everyone is a unique individual with personal values, beliefs, perceptions, culture and understanding of how the world operates. Fragility fractures predominantly, but not exclusively, affect older adults, who formed their opinions, values and beliefs in a very different society to that of the younger people who provide their care, and there can be a cultural age gap that leads to misunderstanding. Nurses need to understand the world from the perspective of an older person to communicate with them effectively and the sensory impairments and potential cognitive dysfunction that are common in older people add to the complexity of developing therapeutic communication. Understanding the acute needs of a patient with a fragility fracture and its management, as well as the chronic underlying diagnosis of osteoporosis and its treatment and impact, add to the already considerable complexity of being an older person with a set of co-morbid medical conditions and social and psychological intricacies resulting from primary and secondary ageing. Effective communication is two-way and involves ensuring that the messages are understood. Barriers to communication may need to be removed in simple ways such as ensuring glasses, hearing aids, interpreters and visual graphics are used to aid communication along with involvement of family friends, caregivers or advocates who know the patient best.

The success of healthcare delivery is often examined by measuring health status, outcomes, readmission rates, length of stay, complication rates and mortality [8], but these do not necessarily capture the specific contribution of nursing. Length of stay is a misleading measure for success as there are decreased levels of expert nursing care when patients are discharged or transferred too early [9]. Appropriate indicators of the quality of nursing care could include measures such as patient comfort and quality of life, safety outcomes (including healthcare-associated infection, pressure ulcers, falls and drug administration errors) and patient satisfaction [8]. In orthogeriatric care, nurse-sensitive indicators might be developed for; pain, delirium, pressure ulcers/injuries, hydration and nutrition, constipation, prevention of secondary infections and venous thromboembolism (VTE) [5]. 


\subsection{The Unique Contribution of Nursing to Orthogeriatric Patient Outcomes}

Nursing care priorities are the fundamental aspects of nursing care including; comfort, hygiene, pain management, nutrition, hydration, remobilisation and rehabilitation. Evidenced-based nursing can coexist with medical models of care, reducing the risk of developing complications, aiming to reduce the risk of morbidity and mortality, whilst improving recovery, maintaining functional ability and improving patient outcomes and experiences [5].

Nurses often become specialists in specific aspects of healthcare to enable them to focus on providing skilled care based on up-to-date knowledge to a defined group, so they often work in teams with other specialists such as medical practitioners and therapists, collaborating in pathways and sharing evidence across professions. Orthogeriatrics was first used to describe collaboration between the specialties of orthopaedic surgery and geriatric medicine but has come to denote a multidisciplinary approach to the hospital care of patients with fragility fractures that recognises the complex specific needs of this group. The concept of orthogeriatrics recognises the need to understand the holistic healthcare needs of frail, elderly patients with multiple health problems at the same time as working towards the best outcomes following fragility fracture [10].

\subsection{Health Improvement and Health Promotion}

The education of patients for health improvement is often the role of nurses because they are the largest group of healthcare providers, but their actual and potential contribution to the management of chronic disease is underappreciated. The reasons for the prevalence of chronic disease in communities are complex; although there is an enormous body of evidence confirming how it can be avoided and treated, prevalence continues to rise and outcomes remain poor. Many patients are often inadequately informed, do not take responsibility for their condition and do not comply with instructions. Management of osteoporosis as a chronic condition provides an example of this; it is difficult to manage as it is often silent and the treatment can be unpalatable; concordance with oral bisphosphonates is poor, particularly in those patients not managed by ongoing nurse coordinator intervention. Nurses working in all settings play a central role in educating and coaching patients and families towards behaviour change that can positively influence health and healthcare outcomes following fragility fracture. The success of health improvement initiatives is reliant on nurses building trust with patients and families and working with them towards improvements in bone health and other related health domains. Every fall and fracture is an opportunity to prevent the next fall or fracture by MDT collaboration to prevent further falls and manage osteoporosis [11]. Nurses must be empowered to use the time they spend giving care with patients and families to educate them about the cause of fragility fractures and involve patients in developing an individualised plan of care for bone health. 


\subsection{The Nursing Resource}

Providing high-quality nursing care is labour intensive and requires an educated and highly skilled workforce led by experienced leaders of care who can direct a team of nurses who are qualified to plan evidence-based care and manage its delivery alongside well-trained and supervised support workers. Because of an ageing workforce and lack of political will to adequately fund nursing, global availability of enough skilled nurses to provide this fundamental and advanced care is a constant source of concern. It has, however, been demonstrated that an increase in a nurses' workload by one patient, from eight to nine patients per qualified nurse, increases the likelihood of an inpatient dying within 30 days of admission by 7\% [12], indicating "missed care" [13] because of difficulties for nurses in undertaking actions to prevent complications and subsequent death.

\subsection{Education for Orthogeriatric Care}

Competence is a hallmark of professional practice [14] but cannot be achieved if nurses do not have the requisite knowledge and skills to deliver care effectively to specific groups of patients. The benefits of providing highly skilled and specialist nursing of fragility fracture patients by practitioners with advanced, specific education have not been explored, but the development of specialist orthogeriatric nursing education could have a positive impact on patient outcomes [9]. Orthogeriatric nursing is an emerging and highly specialised branch of adult nursing that requires skills in both the care of the older person and the care of the orthopaedic and trauma patient so that the practitioner can bring both sets of skills together and use them to provide expert care to patients following fragility fracture with complex care needs that cannot be met without a deep understanding of how both age and frailty, as well as skeletal fragility and injury, impact on the planning and implementation of care. This requires specialist practice that applies knowledge and skills brought together from both nursing disciplines and knowledge and skills from partner professional specialities. Unfortunately, nurses are not well prepared to look after orthogeriatric patients as they have usually worked in orthopaedic trauma units and been educated for the care of adults with musculoskeletal problems rather than specifically to work with frail older people with complex needs. This can lead to the more complex needs of older people not being met. There is, consequently, an important education and skills gap and, at present, there are limited education resources available to support professional development of specialist orthogeriatric nurses.

\subsection{Summary of Main Points for Learning}

The global shortage of nurses is now chronic and, unless the nursing resource is protected and grows, the potential of nursing to impact on patient outcomes and quality of fragility fracture care for patients with fragility fractures will be unmet [9]. 
"Looking after hip fracture patients well is a lot cheaper than looking after them badly" [15] and without a significant nursing resource to do the "looking after well", care will never be cost effective, and chronic health problems will never be prevented. Nurses are central to the coordination, provision and monitoring of orthogeriatric care.

\subsection{Suggested Further Study}

Write a written reflection on how your learning from this chapter, and the entire book, reflects your current practice.

\subsection{How to Self-Assess Learning}

Share your thoughts about the reflection you have written with your manager, mentor or preceptor and identify the ways in which you can become a more effective orthogeriatric practitioner.

\section{References}

1. Hall C, Ritchie D (2013) What is nursing? Exploring theory and practice, 3rd edn. Sage/ Learning Matters, London

2. International Council of Nurses (ICN) (2002) Definition of nursing. http://www.icn.ch/whowe-are/icn-definition-of-nursing/

3. Cipriano P (2007) Celebrating the art and science of nursing. Am Nurse Today 2(5):8

4. Rankin J, Regan S (2004) Complex needs: the future of social care. Institute for public policy research/Turning Point. http://www.ippr.org/files/images/media/files/publication/2011/05/ Meeting_Complex_Needs_full_1301.pdf?noredirect=1

5. Hertz K, Santy-Tomlinson J (2017) The nursing role. In: Falaschi P, Marsh D (eds) Orthogeriatrics. Springer, Cham, pp 131-144

6. McSherry R (2012) Quality of nursing care. In: McSherry W, McSherry R, Watson R (eds) Care in nursing: principles values and skills. Oxford University Press, Oxford

7. Baillie L, Black S (2015) Professional values in nursing. CRC, London

8. Maben J et al (2012) High quality care metrics for nursing. National Nursing Research Unit, King's College London. http://eprints.soton.ac.uk/346019/1/High-Quality-Care-Metrics-forNursing----Nov-2012.pdf

9. Brent L et al (2018) Nursing care of fragility fracture patients. Injury needs completing when published online (in press)

10. Falaschi P, Marsh D (2017) Orthogeriatrics. Springer, Cham

11. Martin FC (2017) Frailty, sarcopenia, falls and fractures. In: Falaschi P, Marsh D (eds) Orthogeriatrics. Springer, Cham, pp 47-62

12. Aiken L et al (2014) Nurse staffing and education and hospital mortality in nine European countries: a retrospective observational study. Lancet 383(993):1824-1830

13. Recio-Saucedo A et al (2017) What impact does nursing care left undone have on patient outcomes? Review of the literature. J Clin Nurs. https://doi.org/10.1111/jocn.140589

14. Drozd M et al (2007) The inherent components of the orthopaedic nursing role: an exploratory study. J Orthop Nurs 11(1):43-52

15. Sahota O, Currie C (2008) Hip fracture care: all change. Editorial. Age Ageing 37:128-129 
Open Access This chapter is licensed under the terms of the Creative Commons Attribution 4.0 International License (http://creativecommons.org/licenses/by/4.0/), which permits use, sharing, adaptation, distribution and reproduction in any medium or format, as long as you give appropriate credit to the original author(s) and the source, provide a link to the Creative Commons license and indicate if changes were made.

The images or other third party material in this chapter are included in the chapter's Creative Commons license, unless indicated otherwise in a credit line to the material. If material is not included in the chapter's Creative Commons license and your intended use is not permitted by statutory regulation or exceeds the permitted use, you will need to obtain permission directly from the copyright holder.

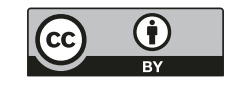

